\title{
Espace de navigation hypermédia
}

The Space of Hypermedia Navigation

\section{Sandro Varano}

\section{OpenEdition}

\section{Journals}

Édition électronique

URL : http://journals.openedition.org/crau/583

DOI : $10.4000 /$ crau. 583

ISSN : 2547-5746

\section{Éditeur}

Éditions du patrimoine

\section{Édition imprimée}

Date de publication : 1 novembre 2012

Pagination : $210-218$

ISBN : 978-2-7577-0108-9

ISSN : 1296-4077

Référence électronique

Sandro Varano, «Espace de navigation hypermédia », Les Cahiers de la recherche architecturale et urbaine [En ligne], 26/27 | 2012, mis en ligne le 01 novembre 2017, consulté le 03 mai 2019. URL : http://journals.openedition.org/crau/583 ; DOI : 10.4000/crau.583 
En exploitant les possibilités offertes par les outils numériques, il s'agit de proposer aux non-experts apprenants, un espace de navigation hypermédia, outil complet de visualisation et d'immersion comme aide à la compréhension des connaissances archéologiques et architecturales. L'espace de navigation présenté met en correspondance des activités d'exploration et de création. Ce travail conduit à un modèle conceptuel applicable à tous types de bâtis.

\section{Espace de navigation
hypermédia}

SANDROVARANO

Ce travail de recherche ${ }^{1} \mathrm{~s}^{\prime}$ inscrit dans le domaine de la restitution archéologique et architecturale. II vise à instruire les publicsapprenants en leur facilitant l'appropriation et la mémorisation de nouvelles connaissances du patrimoine culturel bâti. Les avancées technologiques offrent des situations d'apprentissage dont l'originalité réside dans le mode de navigation tridimensionnel qu'elles proposent : sur le principe de l'interactivité, l'apprenant détermine lui-même son parcours dans un espace hypertextuel multimédia, que nous qualifions d'interface hypermédia. Ces technologies interactives, ont finalement révélé des problèmes de désorientation de l'apprenant, et du même coup de "surcharge cognitive ". II semble important d'envisager les différentes productions cartographiques, littéraires, vidéoludiques..., pour la conception d'un système d'apprentissage instructif et communicatif. Les jeux vidéo présentent tous une dimension réflexive et tactique à des degrés variables et sont des occasions pour les joueurs de mettre en situation leurs compétences dans un environnement sémiologique, narratif et interactif. Les

1. Thèse de doctorat, spécialité Sciences de l'architecture, sous la direction de Jean-Claude Bignon, soutenue le 17 septembre 2010 à I'Institut national polytechnique de Lorraine, Centre de recherche en architecture et ingénierie, MAP CRAI UMR $n^{\circ}$ 694, École nationale supérieure d'architecture de Nancy. Titre: Proposition d'un espace de navigation hypermédia fondé sur des parcours heuristiques comme aide à la compréhension du patrimoine culturel bâti. Spasm : système de parcours d'apprentissage, de scénarisation et de mémorisation. 
recherches en sémiotique croisent aujourd'hui celles des sciences cognitives. Motivation, apprentissage et mémorisation seront traités conjointement avec les technologies de l'information et de la connaissance.

Dans une approche sémio-cognitive, le travail mené correspond à la proposition d'un espace de navigation hypermédia apte à rendre compte de la complexité, tant formelle qu'historique, du patrimoine archéologique et architectural auprès des publics-apprenants. Notre première hypothèse de proposition consistera à établir une trame d'apprentissage, en superposant un parcours topographique et un parcours cognitif. Nous présupposons que cette structure à double couche peut améliorer l'apprentissage par l'intermédiaire des chemins (de l'information et de la connaissance) qu'elle offre et par sa capacité à encourager une attitude ludique. Notre deuxième hypothèse de proposition consistera à établir un " fil narratif » entre les différentes unités d'apprentissage, calqué sur les parcours topographique et cognitif. Nous présupposons que ce " fil sémantique » est capable de tisser des liens significatifs pour l'apprenant, en vue de construire un discours cohérent. Notre troisième et dernière hypothèse de proposition consistera à matérialiser la carte mentale de l'apprenant pour créer une carte mémorielle. Nous présupposons que cette carte, basée sur la métaphore, permet à l'apprenant de s'orienter, et qu'elle assiste sa re-présentation, sa construction de connaissances et à leur mémorisation. Nous souhaitons proposer une modélisation de notre espace de navigation hypermédia. À l'issue de ce travail, le modèle conceptuel représente un espace de navigation générique pouvant être utilisé pour tous types d'édifices architecturaux ou sites archéologiques.

\section{Hypermédias et patrimoine culturel bâti}

Nous pouvons qualifier I'hypermédia comme une extension des deux termes suivants: "hypertexte » et " multimédia ». La notion de parcours est une composante sous-jacente de l'hypertexte ; le passage d'un élément écrit à un autre par l'intermédiaire de liens activables, favorise la construction d'un discours propre au lecteur. Dans un produit hypermédia, I'utilisateur n'interagit pas seulement avec du texte, mais également avec des documents audiovisuels, c'est-à-dire avec des images fixes ou animées et avec du son. La possibilité d'afficher simultanément des informations de natures différentes sur un même support caractérise le multimédia. Finalement, l'hypermédia nous permet de naviguer de manière non-linéaire entre plusieurs informations multimédias (textuelles, visuelles, sonores) grâce à des liens activables que nous appelons des hyperliens. En raison de la diversité des éléments et de la multitude des parcours, les hypermédias offrent des possibilités de navigation interactive variées pour accéder à I'information, nous les résumons en dix points :

- des partitions à l'écran,

- des nœuds et des liens,

- des signes,

- des modes d'exploration,

- des documents multimédias interactifs,

- des temps de lecture,

- des points de vue hétérogènes,

- des visites en cinq dimensions,

- des guides,

- des traces. 


\section{Les chemins de la perdition}

Les hypermédias, associant l'hypertexte et le multimédia, sont en mesure d'offrir une multitude d'interactivités et de présentations, mais impliquent aussi une déperdition de l'information à deux niveaux : l'orientation au sein du parcours, la perception et la compréhension des images. L'hypertexte est de plus en plus associé à la notion $d^{\prime}$ " hypotexte » en raison de l'absence d'horizon de lecture et de la désorientation qu'elle engendre. Le principe de l'hypertexte présente un intérêt certain, dans la mesure où il permet d'envisager une multitude de parcours. Mais le premier danger pour le navigateur est de se perdre; le deuxième danger est d'avoir à gérer un trop grand nombre d'informations, ce qui provoquerait encore une fois une « surcharge cognitive» (Jacquinot, 1996).

\section{La trame topographique et cognitive}

L'assistance à la navigation et à l'acquisition de connaissances passe par l'utilisation de parcours spatiotemporels à caractère stratégique. Le cheminement de l'apprenant est structuré autour de deux types de lieux : les lieux d'information, définissant le chemin de l'information et les lieux de connaissance, définissant le chemin de la connaissance.

Ces deux chemins impliquent une navigation dans un espace organisé et séquencé qui permet de réinvestir constamment les informations et qui, contrairement à l'hypertexte, empêche une déperdition de celles-ci. Chaque point de connaissance est un moment de (re) sémantisation en rapport avec la signification des informations trouvées sur le chemin de l'information.
Pour configurer et mettre en corrélation ces deux chemins d'apprentissage, nous mettons en place un système de deux parcours superposables : nous construisons un parcours cognitif en nous appuyant sur un parcours topographique. Les parcours permettent à l'apprenant de se repérer et de se positionner durant la navigation, aussi bien du point de vue spatial que cognitif.

- le parcours topographique, se situe au niveau des déplacements de l'apprenant et propose des lieux de passages. Le lieu de connaissance est un point de passage structurant le parcours topographique en séquences d'apprentissage. Chacune d'elles possède un seul lieu de connaissance et un nombre variable de lieux d'information.

- le parcours cognitif, se situe au niveau des actions de l'apprenant et propose des types de lieux. Le lieu de connaissance enclenche et résout la séquence, les lieux d'information véhiculent des informations encapsulées dans des objets multimédias interactifs; nous les qualifions $d^{\prime}$ « objets actés» (en référence à l'« image actée »: Weissberg, 1999). Les lieux d'information peuvent être existants au modèle 3D ou rajoutés; par exemple, les Hauts Fourneaux de Pompey en Meurthe-et-Moselle au XIX siècle peuvent être un lieu rajouté à la Tour Eiffel de Paris, la pierre de Rosette au British Museum de Londres peut être un lieu rajouté aidant à la compréhension d'un édifice égyptien. Les interactions possibles en ces lieux doivent permettre à l'apprenant de s'approprier les informations de "niveau zéro " pour les transformer progressivement en informations de "niveau $1^{2}$ » (Bateson, 1977).
2. Le "niveau zéro » de l'apprentissage est la simple réception d'une information. Le " niveau 1 » de l'apprentissage correspond à un changement dans la spécificité de la réponse, à travers une correction des erreurs de choix à l'intérieur d'un ensemble de possibilités » (Bateson, 1977). 
Ces deux parcours sont à la fois une réinterprétation de deux types de narrations interactives vidéoludiques, à savoir les narrations dites "topographique " et « algorithmique » (Favre, 2000, cité par Genvo, 2005), et une combinaison de deux types de structures, à savoir la structure réticulaire de l'hypertexte et la structure linéaire d'un texte littéraire. La configuration de notre trame d'apprentissage est un réel atout à l'optimisation de parcours. Par "optimisation», nous entendons que nos parcours auraient la capacité de s'ajuster à des conditions d'apprentissage à chaque fois différentes. En fonction de la nature des informations et des actions possibles en chaque lieu, d'une part, du nombre de lieux (la distance de cheminement) et du type de cheminement (linéaire, réticulaire et mixtes) entre ces lieux, d'autre part, nous pouvons évaluer la difficulté et la durée qui sera nécessaire pour parcourir les chemins de l'information et de la connaissance. L'« optimisation » est liée aux notions d'effort cognitif et de temps.

\section{Au fil du récit}

À la manière des jeux vidéo, les monuments restitués sont classés par niveaux, possédant une ou plusieurs quêtes qui rend compte d'un aspect du monument (structure, espace, objet...). Le nombre de séquences narratives dans une quête est défini par rapport à l'objectif. Les quêtes proposent plusieurs énigmes à résoudre comme moteurs de base de toute séquence d'apprentissage. Le chemin de la connaissance est un long processus de résolution d'énigmes ; ces dernières sont sources de mystère et entretiennent la motivation qui investie le personnage dans le scénario. Au début de chaque séquence narrative, l'énigme est présentée par l'énoncé descriptif au lieu de connaissance, et va enclencher une démarche indiciaire sur le chemin de l'information. Le nombre de lieux d'information dans une séquence est défini par rapport à l'énigme proposée. Le processus de recherche et de découverte d'indices constitue un cheminement heuristique jusqu'au lieu de connaissance. En retour, le cheminement jusqu'à la résolution de l'énigme est un bon moyen de maintenir le défi (le challenge), le lieu de connaissance fournit un feedback clair et immédiat qui rend compte des performances de l'apprenant. Le scénario a la particularité d'être malléable : il permet d'attribuer diverses caractéristiques aux lieux (focalisations interne ou externe, graphisme réaliste ou non, types d'actions...), ce qui permettra à l'apprenant de distinguer les lieux de connaissance des lieux d'information, car, selon qu'il soit dans l'un ou dans l'autre, il développera des stratégies cognitives différentes, et de différencier les lieux rajoutés des lieux existants, pour ne pas mélanger les « images fausses » et les « images vraies » du monument.

\section{Matérialiser la carte mentale de l'apprenant ${ }^{3}$}

L'objectif est de concevoir une carte mémorielle comme représentation visuelle de l'espace de navigation et comme support de représentations. La carte mémorielle de l'apprenant :

- est utilisée comme outil de repérage et de guidage,

- permet de représenter et de structurer des idées,

- assiste la mémorisation des connaissances.

D’un point de vue sémio-cognitif, la carte mémorielle est support de mémoire pour trois raisons :
3. Jacques Perriault (1994), en se basant sur les recherches de Patricia Greenfield (1994), proposait déjà dans une perspective éducative, de matérialiser les cartes mentales pour les fournir au sujet en tant qu'outil de guidage. 
- I'usage des métaphores de mémoire basées sur "les lieux et les images ${ }^{4}$ " permet la mémorisation des lieux du monument et de leur contenu (Yates, 1987). Les lieux (" loci ») sont les captures d'écran (une photo du lieu), les images (" imagines») sont les informations et les annotations que l'apprenant y dépose ;

- le recours à la " cognition externe ${ }^{5}$ " et à la construction des connaissances permet d'augmenter leur appropriation et leur mémorisation :

- la conservation des traces, révélatrices d'une pratique créatrice, constitue un support de mémoire qui nous préserve de l'oubli et un objet à exploiter par les institutions culturelles (savoir comment les gens apprennent, ...).

La carte mémorielle de l'apprenant prend forme par ajouts successifs des lieux photographiés pendant l'exploration 3D. Elle passe d'une représentation plane bidimensionnelle à une représentation tridimensionnelle selon deux manières : par un effet de pliage et par un déploiement spatial, ce qui permet de représenter les raccourcis entre les pages mémorielles et de situer les pages mémorielles par rapport aux lieux dans le monument (fig. 1).

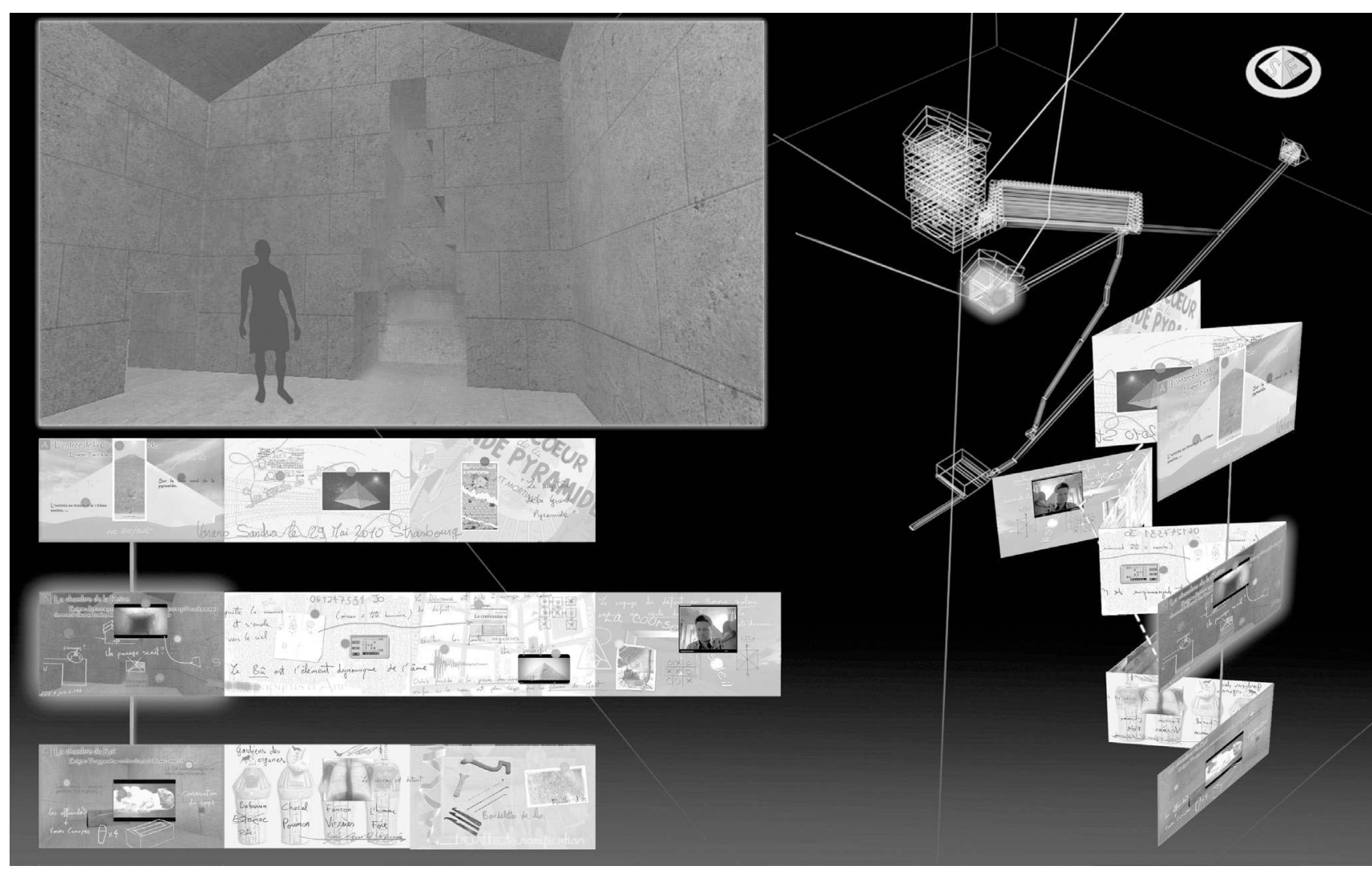

Fig. 1. La lecture simultanée des fenêtres juxtaposées à l'écran ${ }^{6}$. 
Une information, lorsqu'elle est récupérée par une page mémorielle, est rattachée à son connecteur (une " punaise » ou un « aimant » qui lie l'information à la page mémorielle) ; à tout moment, l'apprenant a la possibilité d'y adjoindre des annotations, des croquis... Le deuxième processus de conversion (l'apprentissage de " niveau $2^{7}$ ", Bateson, 1977), démarre directement sur la carte mémorielle. Les informations récupérées sur les pages mémorielles constituent des indices. Les prises de notes supplémentaires aideront l'apprenant à les mettre en corrélation. Lorsque tous les connecteurs sur la page mémorielle du lieu de connaissance sont renseignés, I'apprenant possède une connaissance sur le lieu qui détient l'énigme. La carte mémorielle est un journal identique aux récits illustrés lors des voyages entrepris par les explorateurs et écrivains, eux-mêmes en quête de savoir.

\section{Le modèle conceptuel}

À partir des concepts abordés, nous proposons un modèle conceptuel $\left(U \mathrm{LL}^{8}\right)$ de notre espace de navigation (fig. 2). Dans le texte qui suit, les termes en gras signifient les entités, les termes en italique signifient leurs propriétés et les termes soulignés signifient les relations.

L'apprenant est une personne du public, d'âge variable, impliquée dans un processus d'apprentissage. Nous distinguons plusieurs profils d'apprenants et plusieurs situations d'apprentissage (des élèves en salle de classe, des visiteurs sur une borne muséale, des habitants sur cédérom...). Durant le processus d'apprentissage, l'apprenant explore un seul monument à la fois, puis crée parallèlement sa carte mémorielle (une fois la première capture d'écran déclenchée).
Le monument appartient au patrimoine culturel bâti existant ou non. II se caractérise par sa période historique, son emplacement géographique et son style architectural (gothique, baroque...). II correspond à un niveau qui possède une ou plusieurs quêtes et peut être visité par un apprenant à la fois (dans une perspective éducative, nous pourrions imaginer que les élèves d'une salle de classe explorent le même monument en réseau, c'est-à-dire que les avatars peuvent se croiser dans le monument et sont en compétition ou en collaboration).

La quête correspond à un aspect d'un monument (la structure, les objets, la vie des personnages...). Une fois la quête choisie par l'apprenant, elle présente des options de démarrage : difficulté (facile, moyen, difficile), temps d'apprentissage et nombre de lieux, et propose des types de cheminement (linéaire, réticulaire, mixtes). L'énoncé narratif introduit la quête et annonce l'objectif. Elle possède une ou des séquences d'apprentissage (ou séquences narratives) et une seule carte mémorielle.

Les séquences d'apprentissage (ou narratives) appartiennent à une seule quête à la fois, et sont classées selon la chronologie des faits historiques du monument (séquence d'apprentissage $A$, séquence $d^{\prime}$ apprentissage $B$...). Dans une séquence, nous distinguons les chemins de l'information, de la connaissance et les raccourcis, c'est-à-dire les nouveaux chemins à découvrir par l'apprenant, qui se situent entre les lieux d'une même séquence ou de séquences différentes. Chaque séquence possède un seul lieu de connaissance et au moins un lieu d'information.
4. Dans son livre intitulé L'art de la mémoire, Frances Amelia Yates tente de reconstituer I'histoire de la mémorisation fondée sur des " images » et des « lieux» (1987).

5. Nous passons de la « représentation interne » (image mentale) à la

" représentation externe » et inversement. Selon Zhang et Norman (1994), plus nous disposons d'informations externes, plus nous résolvons facilement le problème posé, plus nous mémorisons.
6. "Blake et Mortimer » (séquence A) est une page mémorielle provenant de Edgar P. Jacobs (1955) ; « La salle de momification » (séquence $C$ ) et "Les tracés du prêtre » (séquence $A$ ) sont des pages mémorielles provenant de Macaulay (2006) ; «Un parcours semé d'embuches » (séquence B) est une page mémorielle inspirée du film Cube 2: Hypercube de Andrzej Sekula (2002), Ghost Logic, Lions Gate Films.
7. Le « niveau 2 » de l'apprentissage opère un changement supplémentaire pour « apprendre à apprendre » (Bateson, 1977).

8. Unified Modeling Language : langage de modélisation unifié. 
La carte mémorielle est créée au fur et à mesure des événements déclenchés par l'apprenant. Son nom est identique à celui de la quête et met à disposition des outils de texte et de dessin. La carte mémorielle appartient à une seule quête à la fois, mais plusieurs cartes mémorielles peuvent être créées si la même quête est choisie par plusieurs apprenants au même moment. La carte mémorielle passe en 3D par pliage et par déploiement, elle possède des pages mémorielles pour les lieux de connaissance et pour les lieux d'information.

Le lieu de connaissance n'appartient qu'à une seule séquence d'apprentissage dans la même quête (par contre, le même lieu de connaissance peut être utilisé dans des quêtes différentes). Son nom est celui du lieu dans le monument et sa localisation est toujours dans



Fig. 2. Modèle conceptuel de I'espace de navigation hypermédia. 
le monument exploré. La focalisation externe permet une exploration 3D sous forme d'avatar, le graphisme réaliste permet de montrer le monument tel qu'il était ou est dans la réalité. La première action (capture écran) génère une page mémorielle du lieu et débloque les portails qui téléportent vers les lieux d'information. Le lieu de connaissance possède une énigme qui sera résolue suite à la réAction finale en ce lieu.

L'énigme est annoncée par la page mémorielle du lieu de connaissance. Un énoncé descriptif la présente. La connaissance est la clé de l'énigme.

Le lieu d'information n'appartient qu'à une seule séquence d'apprentissage dans la même quête (par contre, le même lieu d'information peut être utilisé dans des quêtes différentes). II a un nom et sa localisation est soit interne au monument (avec focalisation externe et graphisme réaliste), soit externe au monument (avec focalisation interne et graphisme non réaliste). Les lieux d'information sont classés par types d'activités (fabrication, voyage, lecture...), et possèdent un ou des objets actés. Dans ces lieux, une zone d'action génère une page mémorielle, ou affiche les objets actés (dans les lieux d'information existants du monument uniquement).

L'objet acté appartient à un seul lieu d'information à la fois. Selon sa nature multimédia et le type d'interaction, l'objet est manipulé et transformé à l'écran en focalisation interne pour construire une information de niveau I, c'est-à-dire un indice.

L'indice délivré par l'objet acté a plusieurs modes de présentation : selon sa nature multimédia, il peut être lu, écouté, vu... L'indice est lié à un seul connecteur qui se trouve sur la page mémorielle du lieu d'information en question.

La page mémorielle porte le nom du lieu auquel elle est rattachée. Des liens 3D entre les pages mémorielles représentent les raccourcis (les chemins cachés). Des annotations sont prises sur les pages par l'apprenant. Il y a deux types de pages mémorielles : une pour les lieux d'information, une pour les lieux de connaissance qui annonce l'énigme et permet des déplacements rapides vers le modèle 3D. Les deux types possèdent des connecteurs.

Le connecteur d'information affiche des indications au passage du curseur de la souris ou du stylet : le mode d'emploi et le but de l'objet acté auquel il correspond. Durant le processus de transformation de I'objet acté, il indique la progression de l'apprenant. Les connecteurs (information) sont destinés à recevoir les indices et sont au même nombre que les objets actés appartenant au lieu d'information. La somme des indices sur une page renseigne les connecteurs de connaissance.

Les connecteurs de connaissance sont au nombre des lieux d'information + 1 (la connaissance), et sont de deux types : ceux qui sont destinés à recevoir les fragments de connaissance, et ceux qui affichent la connaissance finale qui solutionne l'énigme, celle-ci est résolue au lieu de connaissance.

\section{Conclusion}

Dans un souci de restitution et de valorisation du patrimoine culturel bâti auprès des publics-apprenants, déterminer les instruments et les dispositifs technolo- 
giques les mieux adaptés est une tâche essentielle de la médiation culturelle. Aujourd'hui, les outils hypermédias proposent des navigations interactives, qui participent à l'immersion physique et mentale du spectateur/acteur pour lui faire vivre des expériences culturelles inédites. Sur la base des objectifs énoncés en introduction, nous avons proposé un espace de navigation hypermédia qui est composé de plusieurs types de parcours d'apprentissage (topographique, cognitif, scénarisé) superposés et séquencés, impliquant des sous-parcours (les chemins de I'information et de la connaissance). Parallèlement à l'activité d'exploration en temps réel effectuée sur ces parcours, l'apprenant crée sa "carte mémorielle ». Cette dernière utilise la métaphore pour aider l'utilisateur à mémoriser la structure et le contenu de l'espace de navigation; elle soulage la charge cognitive de l'apprenant, et améliore son orientation, sa construction des connaissances et leurs mémorisations. Si nous devions passer aux stades de la réalisation et de I'utilisation, l'aspect de notre espace de navigation se verrait certainement modifié, au moins dans son aspect graphique tel qu'il apparaît à l'écran. Nous avons cherché à constituer des unités d'informations, en reliant significativement leur contenu qui s'est progressivement catégorisé et qui a délimité notre espace de navigation hypermédia. Nous approchons ici de la limite de ce travail essentiellement consacré à I' « utilité » d'un tel espace de navigation, au détriment de I' " utilisabilité », de l'aspect ergonomique (Nielsen, 1994), et en particulier des possibilités d'interventions qui favorisent l'immersion physique de l'apprenant.

\section{B I BLIOGRAPHIE}

- Gregory Bateson, Vers une écologie de l'esprit, tome I, Paris, Seuil, 1977. Traduit de l'américain, Steps to an Ecology of the Mind, San Francisco, Chandler Publishing Company, 1972.

- Julien Favre, «Fiction interactive, quels formats? ", Les Nouveaux Dossiers de l'Audiovisuel, Quels contenus pour Internet?, n 92, juillet-août 2000.

- Sébastien Genvo, «Transmédialité de la narration vidéoludique : quels outils d'analyse? "Comparaison, Peter Lang, 2002, 2, p. 103-112, 2005. Disponible en ligne : http://www.ludologique. com/publis/articles_en_ligne.html.

- P. Mark Greenfield, « Video games as cultural artefacts ». Journal of Applied developmental Psychology, vol. 15, $\mathrm{n}^{\circ} 1$, janvier-mars 1994, p. 3-12.

- Edgar P. Jacobs, « Le mystère de la grande Pyramide ", Les aventures de Blake et Mortimer, tome 2, Paris, Dargaud, 1955, p. 54.

- Geneviève Jacquinot, "Les NTIC : écrans du savoir ou écrans au savoir ", dans Yves Chevalier (dir.), Ateliers 9/1996, «Outils multimédias et stratégies d'apprentissage du FLE ", Cahiers de la Maison de la recherche, tome 1, Lille, université Charles-deGaulle Lille 3, 1996.

- David Macaulay, Naissance d'une pyramide. Archimède, Paris, l'École des loisirs, 2006.
- Jakob Nielsen, Usability Engineering, Cambridge, AP Professional, 1994.

- Jean-Louis Weissberg, Présence à distance. Déplacements virtuels et réseaux numériques. Pourquoi nous ne croyons plus à la télévision. Paris, L'Harmattan, 1999.

- Frances Amelia Yates, L'art de la mémoire, Paris, Éditions Gallimard, traduit de l'anglais par Daniel Arasse, 1987. [The Art of Memory, Londres, Pimlico 1966].

- Jiajie Zhang, Donald A. Norman, "Representations in Distributed Cognitive Tasks ", Cognitive Science, vol. 18, n 1, 1994, p. 87-122. 